OnLine Journal of Biological Sciences 10 (2): 109-113, 2010

ISSN 1608-4217

(C) 2010 Science Publications

\title{
Susceptibility of Candida Species Isolated From HIV Infected and Newborn Candidaemia Patients to Amphotericin B
}

\author{
Saikat Basu, Debasrita Chakraborty and Satadal Das \\ Department of Microbiology and Serology, \\ Peerless Hospital and BK Roy Research Centre, \\ 76 Satyen Roy Road, Kolkata-700094, India
}

\begin{abstract}
Problem statement: HIV infected and neonatal candidaemia is a serious condition where proper selection of the antifungal agent is the key factor in the prognosis of the disease. Thus in this study susceptibility of Candida species to Amphotericin B was explored in such patients. Approach: Forty different Candida isolates obtained from blood culture of such candidaemia cases were tested for Amphotericin B susceptibility by disc diffusion method and by microdilution method. Results: There was a good correlation between low MIC values and large inhibition zones among most isolates as expected, particularly with $24 \mathrm{~h}$ reading. Several outlier data were also observed during the study. After analysis it was found that $24 \mathrm{~h}$ microdilution method results were better for interpretation of susceptibility results. Conclusion: Microdilution method is the reliable method for susceptibility testing of Candida isolates from HIV infected and neonatal candidaemia patients to Amphotericin B.
\end{abstract}

Key words: Candidaemia, disc diffusion, microdilution

\section{INTRODUCTION}

Candidiasis is a common opportunistic infection in immunocompromised hosts and candidaemia causes significant morbidity and mortality in this group of patients (Richards et al., 1999). In recent past, lifethreatening mycoses has been increasing in individuals with compromised immune system and there is a dramatic change in the incidence of different Candida spp. in candidiasis. During last five years (Nguyen et al., 1996). In general Candida albicans is the most abundantly isolated Candida spp. implicated in fungal infections. However, in South East Asian countries incidence of Candida albicans infection had gradually been decreased, while incidence of Candida tropicalis infection was almost equivalently increased during the same period, most of which were fluconazole resistant (Chakrabarty et al., 1999). Antifungal agents currently available for clinical use belong to three major classes: Polyenes, azoles and 5-flucytosine (Martin et al., 1992). Optimally, antifungals should exhibit selective toxicity; they should be able to inhibit the growth of the fungus without adversely affecting the host. It has been noticed that emergence of resistant strains of Candida spp. is due to extensive use of fluconazole as prophylactic treatment in immunocompromised patients (Rattan, 1999).
Even today, Amphotericin B is the gold standard medicine for treating invasive mycoses. It is concentrated primarily in liver and spleen; lesser amounts are accumulated in kidneys and lungs. The primary tissue reservoirs elute the drug back into the blood as plasma levels of the drug fall (Baginski and Czub, 2009). Amphotericin B is selectively taken up into the reticuloendothelial system and concentrated in the reservoir organs. Lipid-rich particles with Amphotericin B are also ingested by phagocytic monocytes; this process helps in targeting the drug to sites of infection or inflammation (Jill and Richard, 2002; Czumb and Baginksi, 2009). In vitro, Amphotericin B demonstrated potent and broadspectrum fungicidal activity against clinically relevant fungi including Candida spp.

The M27-A2, NCCLS, USA (Second Edition) is a "reference" standard protocol for susceptibility testing of Candida spp. It is being developed through a consensus process to facilitate the agreement among laboratories in measuring the susceptibility of yeasts to antifungal agents. The NCCLS method for in vitro susceptibility testing is essential for standardization and to improve inter-laboratory reproducibility, it is difficult to use for all organisms or for routine use in clinical laboratories (National Committee for Clinical Laboratory Standards, 2002). Alternative methods such

Corresponding Author: Satadal Das, Department of Microbiology and Serology, Peerless Hospital and BK Roy Research Centre, 76 Satyen Roy Road, Kolkata-700034, India Tel: +91- 09831092130/033-2462 2394 Fax: 91(33) 24620766 
as the agar-based Disc Diffusion (DD) method are preferable because they are simple and cost effective.

The disease candidaemia is diagnosed when there is isolation of Candida spp. from one or more blood specimens (Edmond et al., 1999). Although oropharyngeal candidiasis being the most common fungal infection affecting patients with HIV and AIDS, candidaemia is most frequent in HIV infected adults particularly with borderline-stage AIDS (CD4+ counts $<50$ cells $\mathrm{mm}^{-3}$ ) and after extensive prior therapy with Amphotericin B. The frequent use of azole in HIV-infected patients compels Amphotericin $\mathrm{B}$ to be used as the first line antifungal agent when candidaemia sets in (Abgrall et al., 2001).

Thus in this study, the applicability of the DD method for testing the susceptibility of Candida species to Amphotericin B was compared with the results of the broth microdilution method of the NCCLS (M-27-A2, NCCLS, USA) for Candida spp. strains isolated from blood of HIV-infected patients and some neonates, the two most susceptible groups for candida infection (Cuenca-Estrella et al., 2002).

\section{MATERIALS AND METHODS}

Candida spp. Isolates from blood culture: All Candida spp. were isolated from candidaemia patients with HIV infection (65\%) and from neonates (35\%). Blood cultures of patients were done in automated blood culture system (Becton Dickinson, BD, BACTEC 9050) in MYCO/F-Lytic bottles. A total of 40 clinical Candida isolates and 3 international control strains comprising of Candida albicans (8), Candida tropicalis (18), Candida glabrata (10) and Candida krusei (4) were taken to study their antifungal susceptibility.

All the isolates were stored in cryogenic vials at $-70^{\circ} \mathrm{C}$ as recommended (Espinel-Ingroff et al., 2004). Species identification were done by germ-tube test, colonial study on Corn Meal Agar (CMA) with 1\% Tween 80/trypan blue, on candida chromogenic media and by carbohydrate assimilation, fermentation tests.

Two types of susceptibility testing-DD method: This test was done using Amphotericin B discs purchased from Himedia, India manufactured according to the $M$ 44-A, NCCLS, USA guidelines (National Committee for Clinical Laboratory Standards, 2004). "Method for Antifungal Disc Diffusion Susceptibility Testing of Yeasts: Propose Guideline M44-A.NCCLS, Wayne, PA., USA)". The drug concentration of the discs was 20 $\mathrm{mcg} \operatorname{disc}^{-1}$. According to the guidelines, the medium required for the disc diffusion test was Mueller-Hinton Agar with $2 \%$ Glucose and $0.5 \mathrm{mcg} \mathrm{mL}^{-1}$ Methylene
Blue Dye (GMB) Medium. Zone diameters (in $\mathrm{mm}$ ) for the zone of complete inhibition were determined after 24 and $48 \mathrm{~h}$ of incubation at $35^{\circ} \mathrm{C}$ and compared with MICs determined by the M27-A2, NCCLS, USA (Second Edition) MD method. All the tested isolates showed good growth rates under these conditions which is a mandatory requirement under this protocol (Lozano-Chiu et al., 1999).

Microdilution method: The antifungal stock solutions were made of standard antifungal powder of Amphotericin B (Obtained from Himedia, India). Its generic name was Amphotericin (Molecular formula$\mathrm{C}_{47} \mathrm{H}_{73} \mathrm{NO}_{17}$ and Molecular weight-924.1) with potency of $750 \mathrm{mcg} \mathrm{mg}^{-1}$. The inoculum suspensions were prepared as instructed in M27-A2, NCCLS, USA (Approved Standard Second Edition) (National Committee for Clinical Laboratory Standards, 2002). "Method for Antifungal Disc Diffusion Susceptibility Testing of Yeasts: Propose Guideline M44-A.NCCLS, Wayne, PA., USA)". Final concentrations of Amphotericin B were 0.0313-16 mcg mL $\mathrm{mL}^{-1}$ (The concentrations were $0.0313,0.0625,0.125,0.25,0.5$, 1.0, 2.0, 4.0, 8.0 and $16.0 \mathrm{mcg} \mathrm{mL}^{-1}$ ). To dilute the stock solution DMSO was used. These microwells were incubated at $35^{\circ} \mathrm{C}$ and MIC endpoints were determined after 24-48 h. The MICs of Amphotericin B were read by Thermo multiskan reader (Vantaa, Finland) as the lowest concentration that caused a significant diminution of growth compared with the control growth well. The medium used in this test was RPMI 1640, the endpoint was considered as a prominent decrease in turbidity corresponding to $\sim 50 \%$ inhibition in growth as determined spectrophotometrically (Bannatyne and Cheung, 1977; Rodriguez-Tudela et al., 1996). It is demonstrated that the most reliable testing criteria for Candida are the use of RPMI broth and a prominent inhibition MIC endpoint. The two types of MICs were determined in this study, these were $\mathrm{MIC}_{50}$ (MIC that inhibits $50 \%$ of the study strain) and $\mathrm{MIC}_{90}$ (MIC that inhibits $90 \%$ of the study strain).

For both methods an arbitrary break point had to be established. Isolates with Diameter inhibition Zone (ZD) of $<16 \mathrm{~mm}$ and MICs $\geq 16 \mathrm{mcg} \mathrm{mL}^{-1}$ were considered to have high MIC values and those isolates with MICs of $<16 \mathrm{mcg} \mathrm{mL}^{-1}$ and Diameter inhibition Zone (ZD) $\geq 16 \mathrm{~mm}$ were considered to have low MIC values.

For quality control three international strains were used-Candida albicans (ATCC 10231), Candida tropicalis (ATCC 750), Candida krusei (ATCC 6258) in every batch tested for antifungal susceptibility (National Committee for Clinical 
Laboratory Standards, 2002; 2004). "Method for Antifungal Disc Diffusion Susceptibility Testing of Yeasts: ProposeGuidelineM44-A.NCCLS, Wayne, PA., USA)".

Statistical analysis of this study: Arithmetic Mean (AMs) and MIC ranges were calculated in each genusspecies combination to evaluate the comparative study between the disc diffusion and broth microdilution methods. The Mean, Standard deviation, Standard error of Mean were calculated in the data sets of different types of variables. As in this method paired data were obtained, so "paired t tests" of every possible combination were analyzed. The linear regression analysis was done in between the DD method and the MD method of antifungal susceptibility testing. Statistical analysis was calculated using Graph Pad and Vassar software.

\section{RESULTS}

There was no significant difference of susceptibility zones by DD method measured at 24 and $48 \mathrm{~h}$ (Table 1). Out of four species, Candida albicans and Candida glabrata showed high susceptibility zones (AM $\geq 18 \mathrm{~mm}$ ) but Candida tropicalis and Candida krusei showed smaller zones (AM <13 mm) in the DD method (Table 2 and 3).

Similarly, AM and ranges of various Candida species studied by micro dilution method was expressed as $\mathrm{MIC}_{50}$. (MIC that inhibits $50 \%$ of study strains), $\mathrm{MIC}_{90}$ (MIC that inhibits $90 \%$ of study strains) and their ranges are given in Table 2 and 3 . In this study Amphotericin B exhibited high antifungal activity against all tested Candida species $\left(\mathrm{IC}_{50} \leq 2 \mathrm{mcg} \mathrm{mL}^{-1}\right.$ in most cases) by the micro dilution method and in this method all species have showed high sensitivity ranges compared to DD method.

Paired ' $t$ ' test value in between $\mathrm{MIC}_{50}$ and $\mathrm{MIC}_{90}$ in 24 h: The two tailed $P$ value was less than 0.0001 . By conventional criteria, this difference was considered to be extremely statistically significant. The standard error of difference was 0.168 .

Table 1: Susceptibility testing results determined by disc diffusion (AM and range) of Amphotericin B

\begin{tabular}{llll}
\hline $\begin{array}{l}\text { Species } \\
\text { (No. of isolates) }\end{array}$ & $\begin{array}{l}\text { Incubation } \\
\text { period }(\mathrm{h})\end{array}$ & $\begin{array}{l}\text { Inhibition } \\
\text { zone }(\mathrm{mm})\end{array}$ & $\begin{array}{l}\text { AM } \\
\text { (range) }\end{array}$ \\
\hline Candida albicans $(8)$ & 24 & 18 & $16-21$ \\
& 48 & 18 & $17-22$ \\
Candida tropicalis $(18)$ & 24 & 11 & $10-13$ \\
& 48 & 11 & $11-14$ \\
Candida glabrata $(10)$ & 24 & 21 & $18-22$ \\
& 48 & 21 & $19-23$ \\
Candida krusei $(4)$ & 24 & 11 & $8-14$ \\
& 48 & 11 & $9-15$ \\
Total (40) & 24 & 15 & $8-21$ \\
& 48 & 15 & $9-23$ \\
\hline
\end{tabular}

AM: Arithmetic Mean
Paired ' $t$ ' test value in between $\mathrm{MIC}_{50}$ and $\mathrm{MIC}_{90}$ in 48 h: The two tailed $P$ value was less than 0.0001. By conventional criteria, this difference was considered to be extremely statistically significant. The standard error of difference was 0.210. A good correlation was observed between low MIC values and large inhibition zones for most of the organism tested as expected. The correlation was better in between results of MD 24/DD $24 \mathrm{~h}$ than results of MD 48/DD $24 \mathrm{~h}(\mathrm{r}=-0.334$ and $\mathrm{r}=-0.270$, respectively) (Fig. 1a and b). There were some aberrant results with few isolates having low MIC ranges after 48 $\mathrm{h}$ of incubation shown small inhibition zones after $24 \mathrm{~h}$ and having high MIC ranges after $24 \mathrm{~h}$ of incubation but showing high inhibition zones after $48 \mathrm{~h}$.

Thus it was found that MD is a reliable method for susceptibility testing of Candida isolates to Amphotericin B in comparison to DD susceptibility testing. Most isolates with MIC sec $\leq 2 \mathrm{mcg} \mathrm{mL}^{-1}$ had $>16 \mathrm{~mm}$ inhibition zones. However, there were few isolates of $C$. tropicalis, C. glabrata and C. krusei where this was not applicable. These isolates had inhibition zone of $<13 \mathrm{~mm}$ but they had the MIC values of $\leq 0.25 \mathrm{mcg} \mathrm{mL}^{-1}$. On the other hand, few isolates of $C$. glabrata and C. albicans have $>20 \mathrm{~mm}$ zones but have $\geq 2 \mathrm{mcg} \mathrm{mL}^{-1}$ MIC values.

When susceptibility of $24 \mathrm{~h}$ DD was compared with $48 \mathrm{~h}$ MD (Fig. 1b) there were few isolates which had the MIC values of $\leq 0.25 \mathrm{mcg} \mathrm{mL}^{-1}$ but low ZD (<13 mm). The best correlation $(95.5 \%)$ was observed between susceptibility results of MD 24 and DD $48 \mathrm{~h}$. The percentage of false resistance/susceptibility was low in case of results with $24 \mathrm{MD} 48 \mathrm{~h}^{-1} \mathrm{DD}$.

Table 2: The suceptibility of Candida spp. against Amphotericin B determined by micro dilution method $\left(\mathrm{MIC}_{50}, \mathrm{MIC}_{90}\right.$ and range) after 24 and $48 \mathrm{~h}$ of incubation

\begin{tabular}{lllll}
\hline $\begin{array}{l}\text { Species } \\
\text { (No. of isolates) }\end{array}$ & $\begin{array}{l}\text { Incubation } \\
\text { period }(\mathrm{h})\end{array}$ & $\begin{array}{l}\mathrm{MIC}_{50} \\
\left(\mathrm{mcg} \mathrm{ML}^{-1}\right)\end{array}$ & $\begin{array}{l}\mathrm{MIC}_{90} \\
\left(\mathrm{mcg} \mathrm{mL}^{-1}\right)\end{array}$ & $\begin{array}{l}\text { Range } \\
\left(\mathrm{mcg} \mathrm{mL}^{-1}\right)\end{array}$ \\
\hline Candida albicans & 24 & 1.22 & 2.80 & $1.0-4.00$ \\
(8) & 48 & 1.22 & 3.20 & $1.0-4.00$ \\
Candida tropicalis) & 24 & 0.86 & 3.26 & $0.25-4.0$ \\
(18) & 48 & 0.84 & 3.90 & $0.25-4.0$ \\
Candida glabrata & 24 & 0.82 & 2.30 & $0.5-4.00$ \\
(10) & 48 & 0.86 & 2.80 & $0.5-4.00$ \\
Candida krusei $(4)$ & 24 & 1.20 & 3.20 & $0.5-4.00$ \\
(4) & 48 & 1.20 & 3.80 & $0.5-4.00$ \\
Total (40) & 24 & 0.97 & 2.90 & $0.5-4.00$ \\
& 48 & 0.96 & 3.50 & $0.5-4.00$ \\
\hline
\end{tabular}

$\mathrm{MIC}_{50}: 50 \%$ inhibition of growth; $\mathrm{MIC}_{90}: 90 \%$ inhibition of growth; AM: Arithmetic Mean

Table 3: Susceptibility testing results after 24 and $48 \mathrm{~h}$ (Mean $\pm \mathrm{SD} \pm$ SEM)

\begin{tabular}{lllll}
\hline MIC and DD types & N (Total no.) & Mean & SD & SEM \\
\hline $\mathrm{MIC}_{50}$ in $24 \mathrm{~h}$ & 43 & 0.9712 & 0.8777 & 0.1338 \\
$\mathrm{MIC}_{90}$ in $24 \mathrm{~h}$ & 43 & 2.9070 & 1.3419 & 0.2046 \\
$\mathrm{MIC}_{50}$ in $48 \mathrm{~h}$ & 43 & 0.9653 & 0.8817 & 0.1345 \\
$\mathrm{MIC}_{90}$ in $48 \mathrm{~h}$ & 43 & 3.5349 & 1.7368 & 0.2649 \\
$\mathrm{DD}$ in $24 \mathrm{~h}$ & 43 & 14.650 & 4.1100 & 0.6300 \\
$\mathrm{DD}$ in $48 \mathrm{~h}$ & 43 & 15.600 & 4.0900 & 0.6200 \\
\hline
\end{tabular}




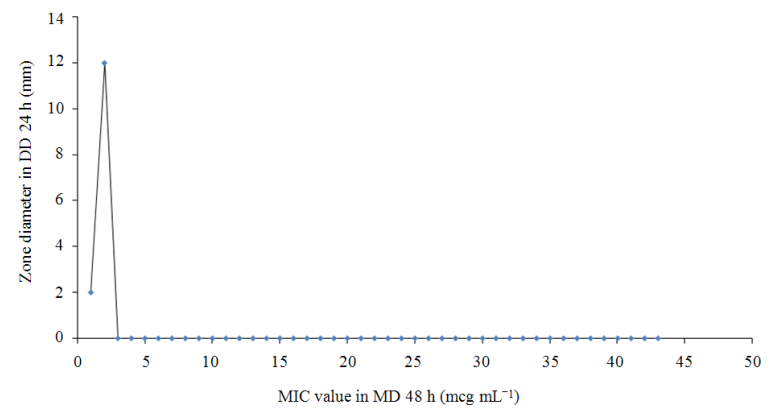

Fig. 1a: Scatterogram showing relationship of $24 \mathrm{~h}$ DD zones of inhibition with $24 \mathrm{~h}$ MIC 90 values. The regression equation was $\mathrm{y}=17.63-1.02 \mathrm{x}$, $\mathrm{r}=-0.334, \mathrm{r}^{2}=0.111$

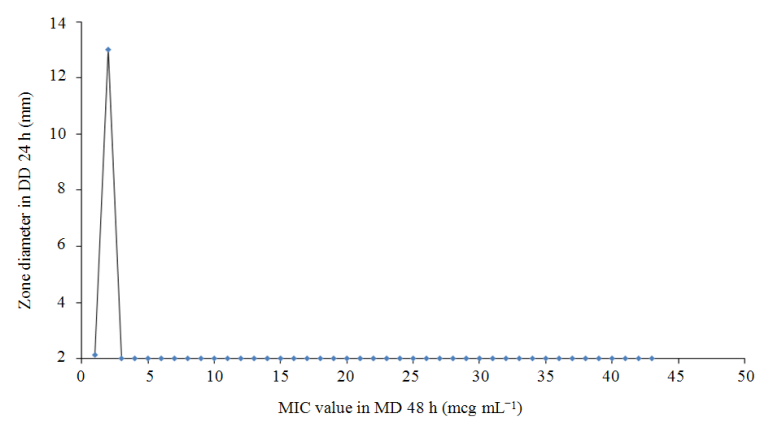

Fig. 1b: Scatterogram showing relationship of $24 \mathrm{~h}$ DD zones of inhibition with $48 \mathrm{~h}$ MIC90 values. The regression equation was $\mathrm{y}=16.91-0.64 \mathrm{x}$, $r=-0.270, r^{2}=0.073$

\section{DISCUSSION}

The ability of the MD method to express the true susceptibility of an individual isolate within $24 \mathrm{~h}$ is its great advantage but it has got a slight disadvantage in relation to its complex preparatory steps. On the contrary, the DD method is less time-consuming and could be a method of choice in a routine clinical laboratory but chance of false positive results are much higher than MD method (Lozano-Chiu et al., 1999). Thus, MD method appears to be superior to DD method.

Our aim to find out a comparative data in relation to an agar-based method and broth MD technique in yeast was thus ended with a conclusive result.

Our conclusion is that the MD method is a very useful and perfect method than the DD method was also supported by the regression analysis (Fig. 1a and b) between MIC values in $24 \mathrm{~h}$ broth MD method with $24 \mathrm{~h}$ susceptibility zone sizes. In the same analysis, the correlation between MIC values in $48 \mathrm{~h}$ broth MD method with $24 \mathrm{~h}$ susceptibility zone sizes was less significant than the previous combination (Ramirez et al., 2006).

\section{CONCLUSION}

The above results suggested that the MD method should be used for testing the susceptibility of Candida spp. isolated from blood to Amphotericin B and as it is possible to get the result in spectrophotometric reading within $24 \mathrm{~h}$, it may help in prevention of many deaths of patients suffering from candidaemia (Menichetti et al., 1994). As most of the candidaemia patients are associated with immunosuppression, it is necessary for the physicians to choose the perfect antifungal drug as soon as possible where MD method appears to be very helpful (Petri et al., 1997). However, there is a need for further studies on Candida strains with high MIC values against Amphotericin B. In developing countries like India it is also important to set up quality control parameters in different clinical laboratories in relation to in vitro susceptibility test by microdilution method which is reliable and better than Disc diffusion method.

\section{ACKNOWLEDGMENT}

We are most grateful to the Managing Director of Peerless Hospital and BK Roy Research Centre for giving us funds and clinical data.

\section{REFERENCES}

Abgrall, S., I. Charreau, V. Joly, J. Bloch and J. Reynes, 2001. Risk factors for esophageal candidiasis in a large cohort of HIV-infected patients treated with nucleoside analogues. Eur. J. Clin. Microbiol. Infect. Dis., 20: 346-349. DOI: 10.1007/s100960100497

Baginski, M. and J. Czub, 2009. Amphotericin B and Its new derivatives-mode of action. Curr. Drug Metab., 10: 459-69. PMID: 19689243

Bannatyne, R.M. and R. Cheung, 1977. Comparative susceptibility of Candida albicans to amphotericin B and amphotericin B methyl ester. Antimicrob. Agents Chemother, 12: 449-450. PMCID: PMC429943

Chakrabarty, A., S. Singh and S. Das, 1999. Changing face of nosocomial candidaemia. Indian J. Med. Microbiol., 17: 160-166.

Cuenca-Estrella, M., W. Lee-Yang and M.A. Ciblak, 2002. Comparative evaluation of NCCLS M27-A and EUCAST broth microdilution procedures for antifungal susceptibility testing of Candida species. Antimicrob. Agents Chemother., 46: 3644-3647. 
Czumb, J. and M. Baginksi, 2009. Amphotericin B and its new derivatives mode of action. Curr. Drug Metab., 10: 459-469. PMID: 19689243

Edmond, M.B., S.E. Wallace and D.K. McClish., 1999. Nosocomial bloodstream infections in United States hospital: A three-year analysis. Clin. Infect. Dis., 29: 23944.

Espinel-Ingroff, A., D. Montero, E. Martin-Mazuelos, 2004. Long-term preservation of fungal isolates in commercially prepared cryogenic microbank vials. J. Clin. Microbiol., 42: 12579. DOI: 10.1007/s 12223-009-0036-3

Jill, A.M. and T. Richard, 2002. liposomal formulation, structure, mechanism of action and pre-clinical experience. J. Antimicrob. Chemother., 49: 21-30.

Lozano-Chiu, M., W.N. Page and L.P. Victor, 1999. Disk diffusion method for determining susceptibilities of Candida spp. to MK-0991. J. Clin. Microbiol., 37: 1625-1627.

Martin, E., P. Parras and M.C. Lozano, 1992. In vitro susceptibility of 245 yeast isolates to amphotericin B, 5-fluorocystosine, ketoconazole, fluconazole and itraconazole. Chemotherapy, 38: 335-339. PMID: 1337508

Menichetti, F., A. Del Favero, P. Martino, G. Bucaneve and A. Micozzi et al., 1994. Preventing fungal infection in neutropenic patients with acute leukemia: Fluconazole compared with oral amphotericin B. The GIMEMA infection program. Ann. Intern. Med., 120: 913-918. PMID: 8172437

National Committee for Clinical Laboratory Standards, 2002. Reference Method for Broth Dilution Antifungal Susceptibility Testing of Yeasts: Approved Standard. M27-A2.NCCLS, Wayne, PA, USA. http://www.clsi.org/source/orders/free/M27S3.pdf
National Committee for Clinical Laboratory Standards, 2004. Method for Antifungal Disc Diffusion Susceptibility Testing of Yeasts: Propose Guideline M44-A.NCCLS, Wayne, PA., USA http://www.clsi.org/source/orders/free/m51-p.pdf

Nguyen, M.H., J.E. Peacock and A.J. Morris, 1996. The changing face of candidemia: Emergence of nonCandida albicans species and antifungal resistance. Am. J. Med., 100: 617-623. DOI: 10.1016/S00029343(95)00010-0

Petri, M.G., J. Konig and H.P. Moecke, 1997. Epidemiology of invasive mycosis in ICU patients: A prospective multicenter study in 435 nonneutropenic patients. Intens. Care Med., 23: 317-325. PMID: 9083235

Ramirez, M., M.C. Serrano, C. Castro, E. Lopez and C. Almeida et al., 2006. Comparative study of disc diffusion and microdilution methods in susceptibility testing of micafungin against Candida species. J. Antimicrob. Chemother, 58: 861-863. DOI: 10.1093/jac/dk1335

Rattan, A., 1999. Antifungal susceptibility testing. Indian J. Med. Microbiol., 17: 125-128. PMID: 16912435

Richards, M.J., J.R. Edwards, D.H. Culver and R.P. Gaynes, 1999. Nosocomial infections in medical intensive care units in the United States. Crit. Care Med., 27: 887-892. DOI: 10.1086/501795

Rodriguez-Tudela, J.L., J. Berenguer, V. MartinezSuarez and R. Sanchez, 1996. Comparison of a spectrophotometric microdilution method with RPMI-2\% glucose with the National Committee for Clinical Laboratory Standards reference macrodilution method M27-P for in vitro susceptibility testing of amphotericin B, flucytosine and fluconazole against Candida albicans. Antimicrob. Agents Chemother., 40: 1998-2003. 